\title{
An explanation for the curious mass loss history of massive stars: From OB stars, through Luminous Blue Variables to Wolf-Rayet stars
}

\author{
H. J. G. L. M. Lamers ${ }^{1,2}$ and T. Nugis ${ }^{3}$ \\ 1 Astronomical Institute, University of Utrecht, Princetonplein 5, 3584CC, Utrecht, The Netherlands \\ 2 SRON Laboratory for Space Research, Utrecht, The Netherlands \\ 3 Tartu Observatory, 61602 Tõravere, Estonia
}

Received18 June 2002 / Accepted 20 September 2002

\begin{abstract}
The stellar winds of massive stars show large changes in mass-loss rates and terminal velocities during their evolution from O-star through the Luminous Blue Variable phase to the Wolf-Rayet phase. The luminosity remains approximately unchanged during these phases. These large changes in wind properties are explained in the context of the radiation driven wind theory, of which we consider four different models. They are due to the evolutionary changes in radius, gravity and surface composition and to the change from optically thin (in continuum) line driven winds to optically thick radiation driven winds.
\end{abstract}

Key words. stars: evolution - stars: mass-loss - stars: Luminous Blue Variables Stars: OB - stars: Wolf-Rayet

\section{Introduction}

In this paper we present an explanation for the drastic changes in mass loss rate and wind velocity during the evolution of massive stars.

The stellar winds of massive stars show a large variation in mass-loss rates and terminal velocities during their evolution from O-star through the Luminous Blue Variable (LBV) phase to the Wolf-Rayet (WR) phase. O-stars have a relatively small mass-loss rate $\left(\dot{M} \simeq 10^{-6}\right.$ to $\left.10^{-5} M_{\odot} \mathrm{yr}^{-1}\right)$ but high wind velocity $\left(v_{\infty} \simeq\right.$ few $\left.10^{3} \mathrm{~km} \mathrm{~s}^{-1}\right)$, LBVs have a high mass-loss rate (few $10^{-5} M_{\odot} \mathrm{yr}^{-1}$ ) but a small wind velocity (few $10^{2} \mathrm{~km} \mathrm{~s}^{-1}$ ) and WR-stars have a high mass-loss rate (few $10^{-5} M_{\odot} \mathrm{yr}^{-1}$ ) and a high wind velocity (few $10^{3} \mathrm{~km} \mathrm{~s}^{-1}$ ). The luminosity remains approximately unchanged during these phases. The question is: what causes these changes in mass-loss rate and in wind velocity?

The mass-loss rates of OB-stars and LBVs during their quiescent phase have been explained in terms of radiation driven winds, where the driving is done by multitudes of spectral lines (Castor et al. 1975; Pauldrach et al. 1986; Vink et al. 2000). Line driven winds are optically thin in their continuum. Nugis \& Lamers (2002, hereafter NL) have shown that the massloss rates of Wolf-Rayet stars can in principle be explained by

Send offprint requests to: H. J. G. L. M. Lamers,

e-mail: lamers@astro.uu.nl optically thick radiation driven winds. In this paper we explain the large changes in $\dot{M}$ and $v_{\infty}$ in terms of transitions between four types of radiation driven wind models, due to changes mainly in the stellar parameters and to a smaller extent also in the surface abundance during the stellar evolution. We discuss the properties of two types of optically thin and two types of optically thick radiation driven wind models, which we then apply to nine characteristic massive stars with increasing evolution stages.

\section{Evolutionary changes of the stellar parameters of massive stars}

The evolution of stars initially more massive than about $50 M_{\odot}$ proceeds as follows (e.g. Maeder \& Meynet 1987). After the main sequence phase the star expands and becomes a blue supergiant with a radius of about $10^{2} R_{\odot}$ and with an enhanced He and $\mathrm{N}$ surface abundance. For a reason that is poorly understood the star becomes an unstable LBV with multiple outbursts (Humphreys \& Davidson 1994; Leitherer 1997). Even during quiesence the mass-loss rates of LBVs are significantly higher than during the main sequence phase but the wind velocity is much lower. After the LBV-phase the star contracts and becomes a WR star with a N-rich (WN-type) and later a C, O-rich (WC-type) surface. The hydrostatic radius of the star is only a few $R_{\odot}$, but the high mass-loss rate produces an optically thick wind with a sonic point radii of about 15 
to $30 R_{\odot}$ for hydrogen-rich WNL-phase and about 1.5 to $10 R_{\odot}$ for the H-poor WNE/WC phase (see NL). Very massive stars $\left(M>60 M_{\odot}\right)$ may skip the LBV phase (Bohannan \& Crowther 1999).

We adopt nine typical massive stars of about the same luminosity and initial mass to characterize the changes in the stellar and wind parameters during the evolution of a massive star. They represent different evolutionary phases. The stars are listed in Table 1 in order of increasing evolutionary stage: one Of-star ( $\zeta$ Pup), one LBV (P Cyg), three stars with spectra in between those of Of and late-WN (HD 151804, HD 152408, HD 152386), three WN-stars (WR 105, WR 136 and WR 139) and one WC-star (WR 111). Notice the large changes in $\dot{M}$ and $v_{\infty}$ and the general increase of the atmospheric $\mathrm{He} / \mathrm{H}$-ratio and of the momentum transfer efficiency, $\eta=\dot{M} v_{\infty} /(L / c)$, with evolution stage. The radii of the four genuine $\mathrm{WN}$ and WC-stars in this table are the radii of the sonic point, derived by $\mathrm{NL}$, and the value of $T_{\mathrm{eff}}$ is at that radius.

\section{Radiation driven wind models}

\subsection{Optically thin line driven winds}

For line driven wind models, which are optically thin in the continuum, the predicted terminal velocity $v_{\infty}$ is

$v_{\infty} \simeq C_{\mathrm{fd}} \sqrt{\alpha /(1-\alpha)} v_{\mathrm{esc}}$

(Castor et al. 1975; Kudritzki et al. 1989), where $\alpha$ is a force multiplier parameter with $\alpha \simeq 0.5$ to 0.7 for hot massive stars of $T_{\text {eff }} \gtrsim 8000 \mathrm{~K}$ and $v_{\text {esc }}$ is the effective escape velocity, i.e. corrected for radiation pressure by electron scattering. Lamers et al. (1995) have shown that $C_{\mathrm{fd}} \sqrt{\alpha /(1-\alpha)} \simeq 2.7$ if $T_{\text {eff }} \gtrsim$ $21000 \mathrm{~K}$ and 1.3 if $10000 \lesssim T_{\text {eff }} \lesssim 21000 \mathrm{~K}$. Observations and theory both show that the mass-loss rate of a line driven wind increases by about a factor 5 and the terminal velocity decreases by about a factor two when the effective temperature of the star drops below about $21000 \mathrm{~K}$ (Lamers et al. 1995; Vink et al. 1999). This is the bi-stability jump, which is due to the change in ionization in the lower wind layers near the sonic point (Vink et al. 1999). Detailed calculations of line driven wind models with multiple scattering by Vink et al. (2001) have shown that the mass-loss rate of galactic OB-stars in $M_{\odot} \mathrm{yr}^{-1}$ is

$$
\begin{aligned}
\log \dot{M}= & -6.86+2.194 \log \left(L / 10^{5}\right)-1.313 \log (M / 30) \\
& +0.933 \log \left(T_{\text {eff }} / 40000\right)-10.92\left\{\log \left(T_{\text {eff }} / 40000\right)\right\}^{2}
\end{aligned}
$$

on the "hot side" of the jump, $T_{\text {eff }} \geq 21000 \mathrm{~K}$, and

$$
\begin{aligned}
\log \dot{M}= & -6.39+2.210 \log \left(L / 10^{5}\right)-1.339 \log (M / 30) \\
& +1.07 \log \left(T_{\text {eff }} / 20000\right)
\end{aligned}
$$

on the "cool side" of the jump, $10000 \leq T_{\text {eff }} \leq 21000 \mathrm{~K}$, with $M$ and $L$ in solar units. We will use Eqs. (1)-(3) to predict $v_{\infty}$ and $\dot{M}$ for line driven winds.

\subsection{Optically thick radiation driven winds}

NL have shown that in optically thick radiation driven winds the opacity has to increase outwards at the sonic point. They showed that

$\dot{M} \approx C \frac{T_{\mathrm{s}}^{4} R_{\mathrm{s}}^{3} v_{\mathrm{s}}}{M}=4.66 \times 10^{-29} \frac{T_{\mathrm{s}}^{4.5} R_{\mathrm{s}}^{3}}{M} \sqrt{\frac{(1+\gamma)}{\mu}}$

in $M_{\odot} \mathrm{yr}^{-1}$, where $R_{\mathrm{s}}\left(\right.$ in $R_{\odot}$ ) and $T_{\mathrm{s}}$ (in $\mathrm{K}$ ) are the radius and temperature at the sonic point, $M$ is in $M_{\odot}, v_{\mathrm{S}}$ is the isothermal sound speed, $\gamma$ is the mean number of free electrons per atom and $\mu$ is the mean atomic weight in atomic mass units. We see that $\dot{M}$ of an optically thick radiation driven wind is proportional to $T_{\mathrm{s}}^{4.5}$. At first sight this might suggest that an arbitrary high mass-loss rate can be reached by moving the sonic point deeper into the star where the optical depth and the temperature are higher. However, this is not the case, because the transition from subsonic to supersonic velocity at the sonic point sets requirements for the opacity and its gradient. The opacity and its gradient at the sonic point are (see NL)

$\chi_{\mathrm{s}} \simeq \frac{4 \pi c G M}{L}$,

$\left(\frac{\mathrm{d} \chi}{\mathrm{d} r}\right)_{\mathrm{s}} \simeq \chi_{\mathrm{s}} \frac{3 v_{\mathrm{s}}^{2}}{G M}=\chi_{\mathrm{s}} \frac{3 a_{1} T_{\mathrm{s}}}{G M}=\frac{12 \pi c a_{1} T_{\mathrm{s}}}{L}>0$,

where $a_{1}=k(\gamma+1) /\left(\mu m_{\mathrm{u}}\right)$. Equations (5) and (6) imply that the transonic transition can only occur in the layers where the opacity increases outwards and where it reaches a value set by the luminosity and mass of the star (Eq. (5)). From the OPALopacity tables (Iglesias \& Rogers 1996) we find that this occurs only in limited temperature regimes where $\chi(T)$ shows a bump. These regimes are in the ranges of $156000 \leq T_{\mathrm{s}} \leq 162000 \mathrm{~K}$, and $37000 \leq T_{\mathrm{s}} \leq 71000 \mathrm{~K}$, where respectively a large and a small Fe-opacity peak occur (see NL). We will use Eq. (4) to predict $\dot{M}$ for optically thick winds. For $v_{\infty}$ we adopt the scaling predicted by the models. We derived from WR models of NL that

$v_{\infty} \simeq(2 \pm 0.5) v_{\mathrm{esc}}^{\mathrm{s}}$

with $v_{\mathrm{esc}}^{\mathrm{s}}$ at the sonic point. We will use this scaling law to estimate $v_{\infty}$ for optically thick radiation driven winds. Notice that $v_{\infty}$ increases with decreasing sonic radius.

\subsection{Four types of wind models}

The description above has shown that radiation driven winds from hot stars come in four types:

(1) line driven winds which are optically thin in the continuum for stars with $T_{\text {eff }} \geq 21000 \mathrm{~K}$, i.e. on the hot side of the bistability jump: "line hot" models. For these winds we adopt Eqs. (1) and (2);

(2) line driven winds for stars with $T_{\mathrm{eff}} \leq 21000 \mathrm{~K}$, i.e. on the cool side of the bi-stability jump: "line cool" models. We adopt the Eqs. (1) and (3) for these models;

(3) "thick cool" continuum driven winds, with the sonic point in the temperature range of $38000<T_{\mathrm{s}}<71000 \mathrm{~K}$, where the 
Table 1. Nine characteristic massive stars.

\begin{tabular}{|c|c|c|c|c|c|c|c|c|c|c|c|}
\hline Name & Type & $\begin{array}{c}\log L \\
L_{\odot}\end{array}$ & $\begin{array}{c}R^{(1)} \\
R_{\odot}\end{array}$ & $\begin{array}{c}T_{\text {eff }} \\
\mathrm{K}\end{array}$ & $\begin{array}{c}M^{(2)} \\
M_{\odot}\end{array}$ & $\begin{array}{c}v_{\mathrm{esc}}{ }^{(3)} \\
\mathrm{km} \mathrm{s}^{-1}\end{array}$ & $N_{\mathrm{He}} / N_{\mathrm{H}}$ & $\begin{array}{c}\dot{M} \\
M_{\odot} \mathrm{yr}^{-1}\end{array}$ & $\begin{array}{c}v_{\infty} \\
\mathrm{km} \mathrm{s}^{-1}\end{array}$ & $\eta$ & Ref. $^{(4)}$ \\
\hline$\zeta$ Pup & O4 If & 6.00 & 19 & 42000 & 70 & 953 & 0.15 & $3 \times 10^{-6}$ & 2250 & 0.32 & PU \\
\hline P Cyg & $\mathrm{B} 1.5 \mathrm{Ia}^{+}$ & 5.86 & 76 & 19300 & 23 & 223 & 0.30 & $2 \times 10^{-5}$ & 210 & 0.28 & PP \\
\hline HD151804 & O8 Iaf & 5.84 & 37 & 26700 & 46 & 581 & 0.25 & $1.2 \times 10^{-5}$ & 1445 & 1.24 & $\mathrm{CB}$ \\
\hline HD152408 & WN9ha & 5.80 & 32 & 27600 & 44 & 648 & 0.67 & $2.4 \times 10^{-5}$ & 995 & 1.79 & $\mathrm{CB}$ \\
\hline HD152386 & WN9ha & 5.82 & 33 & 27000 & 46 & 624 & 0.27 & $2.7 \times 10^{-5}$ & 1650 & 3.45 & $\mathrm{BC}$ \\
\hline WR 105 & WN 9 & 5.81 & 26 & 32100 & 22 & 412 & 0.44 & $2.8 \times 10^{-5}$ & 1200 & 2.6 & NL \\
\hline WR 136 & WN $6 \mathrm{~b}$ & 5.73 & 4.6 & 73000 & 19 & 900 & 1.9 & $6.3 \times 10^{-5}$ & 1600 & 9.2 & NL \\
\hline WR 139 & WN 5 & 5.21 & 2.0 & 82000 & 9.3 & 1129 & 5.0 & $0.9 \times 10^{-5}$ & 1800 & 4.9 & NL \\
\hline WR 111 & WC 5 & 5.31 & 2.3 & 81000 & 10.6 & 1160 & $\infty$ & $1.0 \times 10^{-5}$ & 2415 & 5.8 & NL \\
\hline
\end{tabular}

(1) For WR-stars the mean sonic radius of the models A1 and B1 of NL with the corresponding $T_{\text {eff }}=\left(L /\left(4 \pi \sigma R_{\mathrm{s}}^{2}\right)\right)^{0.25}$ is listed.

(2) Masses of $\mathrm{O}$ and WN9ha-stars were derived from evolutionary models.

(3) $v_{\text {esc }}$ is the effective escape velocity at the radius $R$, corrected for radiation pressure by electron scattering.

(4) PU = Puls et al. (1996); PP = Pauldrach \& Puls (1990); CB = Crowther \& Bohannan (1997); BC = Bohannan \& Crowther (1999);

$\mathrm{NL}=$ Nugis \& Lamers (2002).

small opacity bump occurs (see NL). For these stars we adopt Eqs. (4) and (7) with $T_{\mathrm{s}}=40000$ and $70000 \mathrm{~K}$;

(4) "thick hot" continuum driven winds. For these stars we adopt Eqs. (4) and (7) with $T_{\mathrm{s}} \simeq 160000 \mathrm{~K}$.

\section{Predicted radiation driven mass-loss rates and velocities}

We apply the predictions of the radiation driven wind models to the nine stars. The resulting values of $\dot{M}$ and $v_{\infty}$ are listed in Table 2 for four models: "line cool" or "line hot", "thick cool" with $T_{\mathrm{s}}=40000$ and $70000 \mathrm{~K}$, and "thick hot" with $T_{\mathrm{s}}=160000 \mathrm{~K}$. For the line driven wind model of P Cyg we adopt the predictions for the cool side of the bi-stability jump ("line cool") whereas for the other stars we adopt the "line hot" models.

Comparing the predicted values of $\dot{M}$ and $v_{\infty}$ of the four models with the observed values, we can determine which model fits best. In this comparison we have given more weight to the mass-loss rate than to the terminal velocity, because the predicted $v_{\infty}$ of the line driven models depends on the mass of the star, which is not well known, and $v_{\infty}$ of the optically thick winds is not well predicted by NL models. For the star $\zeta$ Pup the models "line hot" and "thick cool" with $T_{\mathrm{s}}=40 \mathrm{kK}$ predict almost the same mass-loss rates. We adopt the "line hot" model, because this star is considered to be the prototype of a line driven wind (e.g. Pauldrach et al. 1994). In the case of the O8 Iaf star HD $151804 \dot{M}$ is in between the predicted values of the "line hot" and "thick cool" $(40 \mathrm{kK})$ models. The observed value of $v_{\infty}$ agrees better with the "line hot" model. The wind of this star may be of intermediate type. For the other stars the choice of the best fitting model is quite obvious (last column of Table 2). Notice that for the "thick cool" models the observed values of $\dot{M}$ agree better with those predicted for $T_{\mathrm{s}} \simeq 40 \mathrm{kK}$ than for $T_{\mathrm{s}} \simeq 70 \mathrm{kK}$.

\section{Discussion and conclusions}

From the comparison between the predicted and the observed values of $\dot{M}$ and $v_{\infty}$, we can explain the changes in mass-loss rate and wind velocities during the evolution of massive stars in terms of the four wind models.

1. O-stars on the main sequence and shortly thereafter have winds driven by lines on the hot side of the bi-stability jump.

2. The winds of LBVs are radiation driven by lines. The increase in $\dot{M}$ and the decrease in $v_{\infty}$ from O-star to LBV is due to the crossing of the bi-stability jump. (However, not all LBVs become cool enough to reach the bi-stability jump: Leitherer 1997; Lamers 1997)

3. When the star has lost sufficient mass and the atmosphere has been He-enriched sufficiently to contract to the WNL-phase, the wind becomes optically thick and the sonic point moves into the region where the continuum opacity shows a small bump so that it can initiate an optically thick wind. This results in a (small) increase of $\dot{M}$ and a large increase in $v_{\infty}$. The characteristics of the star HD 151804 suggest that the transition from line driven winds to "thick cool" is gradual.

4. When the star looses more mass and the surface becomes H-poor its wind may either stay "thick cool" (at $T_{\mathrm{s}} \simeq$ $40 \mathrm{kK}$ ) or become "thick hot". In the first case the star may appear as a H-poor WN7 or WN8-star (not studied here). In the latter case the star appears as a WNE-star, similar to WR 136 and WR 139. In both cases the star has a high $\dot{M}$ and a high $v_{\infty}$. The transition from a "thick cool" to "thick hot" wind is determined by the variation of $\chi_{\mathrm{s}}$ (see Eq. (5)). During the WNE-phase the $M / L$-ratio increases (Schaerer \& Maeder 1992). When $\chi_{\mathrm{s}}$ increases to values above $\simeq 0.5 \mathrm{~cm}^{2} \mathrm{~g}^{-1}$, the sonic point has to move to high temperature regime, because such a high value of $\chi_{\mathrm{s}}$ is reached only near the main iron opacity peak (NL). 
Table 2. Predictions for line driven (optically thin continuum) and optically thick winds versus observations.

\begin{tabular}{|c|c|c|c|c|c|c|c|c|c|c|}
\hline Star & Type & $\begin{array}{l}\text { thin } \\
\text { line }\end{array}$ & $\begin{array}{c}\text { thick } \\
\text { cool } \\
40 \mathrm{kK}\end{array}$ & $\begin{array}{c}\log \dot{M} \\
\text { thick } \\
\text { cool } \\
70 \mathrm{kK}\end{array}$ & $\begin{array}{c}\left(M_{\odot} \mathrm{yr}^{-1}\right) \\
\text { thick } \\
\text { hot } \\
160 \mathrm{kK}\end{array}$ & obs & $\begin{array}{l}v_{\infty} \\
\text { thin } \\
\text { line }\end{array}$ & $\begin{array}{c}\left(\mathrm{km} \mathrm{s}^{-1}\right) \\
\text { thick }\end{array}$ & obs & $\begin{array}{l}\text { Best } \\
\text { model }\end{array}$ \\
\hline$\zeta$ Pup & O4 If & -5.12 & -5.54 & -4.44 & -2.83 & -5.52 & 2573 & 1906 & 2250 & line hot \\
\hline P Cyg & $\mathrm{B} 1 \mathrm{Ia}^{+}$ & -4.35 & -3.28 & -2.19 & -0.58 & -4.70 & 290 & 446 & 210 & line cool \\
\hline HD 151804 & O8 Iaf & -5.71 & -4.51 & -3.42 & -1.81 & -4.92 & 1569 & 1162 & 1445 & line/thick? \\
\hline HD 152408 & WN9ha & -5.68 & -4.73 & -3.65 & -2.02 & -4.62 & 1750 & 1296 & 955 & thick cool \\
\hline HD 152386 & WN9ha & -5.78 & -4.67 & -3.57 & -1.96 & -4.56 & 1685 & 1248 & 1650 & thick cool \\
\hline WR 105 & WN 9 & -5.09 & -4.68 & -3.59 & -1.97 & -4.55 & 1037 & 824 & 1200 & thick cool \\
\hline WR 136 & WN $6 \mathrm{~b}$ & -5.50 & -6.94 & -5.84 & -4.22 & -4.20 & 2430 & 1800 & 1600 & thick hot \\
\hline WR 139 & WN 5 & -6.50 & -7.73 & -6.64 & -5.03 & -5.05 & 3048 & 2258 & 1800 & thick hot \\
\hline WR 111 & WC 5 & -6.33 & -7.70 & -6.60 & -4.99 & -5.00 & 3132 & 2320 & 2415 & thick hot \\
\hline
\end{tabular}

When the star evolves directly from Of to WNLh both the $M / L$-ratio and $\chi_{\mathrm{s}}$ decrease.

5. It is difficult to predict the dependence of $\dot{M}$ on $L$ for optically thick wind models accurately because $R_{\mathrm{s}}$ and hence also $T_{\text {eff }}\left(R_{\mathrm{S}}\right)$ is not well known. The wind models of NL for WNE/WCE stars predict that $R_{\mathrm{S}} \propto L^{0.7}$ and because $M \propto L^{0.6}$ (Schaerer \& Maeder 1992), it follows that $\dot{M} \propto R_{\mathrm{s}}{ }^{3} / M \propto L^{1.5}$ (Eq. (4)). This dependence agrees well with the empirical relation derived by Nugis \& Lamers (2000) for WN stars. For the WC stars Nugis \& Lamers (2000) found empirically that $\dot{M} \propto L^{0.84}$ but with strong dependence on chemical composition. On the other hand, in a recent study of LMC WC-stars Crowther et al. (A\&A in press) found a strong dependence of $\dot{M}$ on $L\left(\dot{M} \propto L^{1.38}\right)$ which agrees well with our predicted dependence for optically "thick hot" wind models.

6. We find that the changes in $\dot{M}$ and $v_{\infty}$ during the evolution of massive stars are mainly due to changes in the stellar parameters and to a lesser degree to changes in the surface composition.

We have shown that the changes in $\dot{M}$ and $v_{\infty}$ during the evolution of the massive stars from O-star to $\mathrm{WN}$-star are due to the adjustment of the wind to the changing conditions, mainly the $M, L, R$ and surface composition. We have not explained "how" these changes occur. The transition from line driven winds to "thick cool" winds is most likely due to the formation of a bump in the opacity curve when the $\mathrm{He} / \mathrm{H}$ ratio increases. This transition can occur gradually because the temperature of the sonic point of a "line hot" wind overlaps with the range for "thick cool" winds. The transition from "thick cool" to "thick hot" is probably due to the fact that the hydrostatic radius of a WR-star shrinks and the $M / L$-ratio increases when the luminosity of the star decreases (Schaerer \& Maeder 1992). This transition cannot be gradual, because the sonic point temperature in these models is very different, so the wind must be restructured during this transition.

We point out that at present, the optically thick wind models do not provide accurate predictions for $v_{\infty}$, so we adopted an empirical scaling law. However, Schmutz (1997) has shown that there is sufficient driving in the supersonic part of the winds of WR-stars to explain the observed high values of $v_{\infty}$.

Acknowledgements. We thank an unkown referee for positive and constructive comments. This work was supported by the Estonian Science Foundation grant No. 5003. T.N. is grateful to The Netherlands School of Astronomy (NOVA) for a travel grant.

\section{References}

Bohannan, B. A., \& Crowther, P. A. 1999, ApJ, 511, 374

Castor, J. I., Abbott, D. C., \& Klein, R. I. 1975, ApJ, 195, 157

Crowther, P. A., \& Bohannan, B. A. 1997, A\&A, 317, 532

Humphreys, R. M., \& Davidson, K. 1994, PASP, 106, 1025

Iglesias, C. A., \& Rogers, F. J. 1996, ApJ, 464, 943

Kudritzki, R. P., Pauldrach, A. W. A., Puls, J., \& Abbott, D. C. 1989, A\&A, 219, 205

Lamers, H. J. G. L. M. 1997, in Luminous Blue Variables, Massive stars in transition, ed. A. Nota, \& H. J. G. L. M. Lamers, ASP Conf. Ser., 120, 76

Lamers, H. J. G. L. M., Snow, T. P., \& Lindholm, D. 1995, ApJ, 455, 269

Leitherer, C. 1997, in ASP Conf. Ser. 120, ed. A. Nota, \& H. J. G. L. M. Lamers, 58

Maeder, A., \& Meynet, G. 1987, A\&A, 182, 243

Nugis, T., \& Lamers, H. J. G. L. M. 2000, A\&A, 360, 227

Nugis, T., \& Lamers, H. J. G. L. M. 2002, A\&A, 389, 162

Pauldrach, A. W. A., \& Puls, J. 1990, A\&A, 237, 409

Pauldrach, A. W. A., Puls, J., \& Kudritzki, R. P. 1986, A\&A, 164, 86

Pauldrach, A. W. A., Kudritzki, R. P., Puls, J., Butler, K., \& Hunsinger, J. 1994, A\&A, 183, 525

Puls, J., Kudritzki, R. P., Herrero, A., et al. 1996, A\&A, 305, 171

Schaerer, D., \& Maeder, A. 1992, A\&A, 263, 129

Schmutz, W. 1997, A\&A , 321, 268

Vink, J. S., de Koter, A., \& Lamers, H. J. G. L. M. 1999, A\&A, 350, 181

Vink, J. S., de Koter, A., \& Lamers, H. J. G. L. M. 2000, A\&A, 362, 295

Vink, J. S., de Koter, A., \& Lamers, H. J. G. L. M. 2001, A\&A, 369, 574 